\title{
Time-Series Modeling of Multiple Sclerosis Disease Activity: A Promising Window on Disease Progression and Repair Potential?
}

\author{
Dominik S. Meier, ${ }^{*}$ Howard L. Weiner, ${ }^{\dagger}$ and Charles R. G. Guttmann* \\ Center for Neurological Imaging, Departments of *Radiology and ${ }^{\dagger}$ Neurology, Brigham and Women's Hospital, Harvard \\ Medical School, Boston, Massachusetts 02115
}

Summary: This article discusses and reviews advanced forms of serial morphometry in the context of a disease progression model in multiple sclerosis (MS). This model of disease activity distinguishes between overall disease activity and the proportion thereof that becomes permanent damage. This translates into a progression model that features a repair potential, which, when exhausted, marks the conversion or progression from relapsing to progressive disease. The level of repair capacity at a given time determines the rate of progression. Both clinical and MRI variables appear to be in support of such a model. We examine possible MRI markers for this repair capacity, particularly the short-term behavior of new MRI lesions, quantified by methods of time-series analysis- that is, capturing lesion dynamics in the form of MRI intensity change directly, rather than shape or volume change. Lower rates of individual lesion recovery may represent lower repair and greater proximity to a progressive stage. Individuals with low transient lesion turnover appear to undergo more rapid progression and atrophy. Because disease-modifying therapies aim to alter the pathophysiological chain of inflammation, demyelination, and axonal loss, a therapeutic effect may therefore be more readily apparent as a change in lesion dynamics and recovery rate and level, rather than a change in total lesion burden or enhancing lesion number. Key Words: Multiple sclerosis, disease modeling, serial MRI, morphometry, lesion evolution, repair.

\section{THE REPAIR POTENTIAL HYPOTHESIS}

More than $80 \%$ of newly diagnosed cases of clinically definite multiple sclerosis (MS) fall into the relapsingremitting (RRMS) category. A large proportion of RRMS patients eventually convert to a secondary progressive stage (SPMS) within $6-10$ years. ${ }^{1,2}$ The hallmark of this stage is a progressive worsening of disability in the absence of relapses, and a shift from inflammatory to degenerative activity, apparent on MRI as fewer contrast-enhancing lesions and accelerated brain parenchymal atrophy (FIG. 1). The etiology of this progression is not known, but a premise commonly offered is that of a threshold effect and the exhaustion of structural and functional redundancy, leading from in-

Address correspondence and reprint requests to Dominik Meier, $\mathrm{Ph} . D .$, Center for Neurological Imaging, Brigham and Women's Hospital, 221 Longwood Avenue, RF 396, Boston, MA 02115. E-mail: meier@bwh.harvard.edu. flammatory and relapsing to more diffuse and accelerated accrual of damage.

Here we review and discuss this progression model, focusing on the concept of a hypothesized repair potential as a marker for progression. Of particular interest is the possibility of obtaining early MRI markers of this repair potential from advanced forms of serial quantitativ MRI. As new therapies with alternative mechanisms (other than broad or targeted immune suppression) become available to MS patients, specific markers that reliably predict long-term progression early in the disease are becoming increasingly critical.

A growing body of evidence is congruent with a repair potential model: histopathological analyses have revealed a shift from focal inflammatory activity in RRMS to diffuse damage in $\mathrm{SPMS}^{3}$ and overall reduced inflammation in SPMS lesions. ${ }^{4}$ An altered role of inflammation in SPMS is documented also from multiple treatment trials in which inflammation was effectively suppressed but accrual of atrophy remained unaltered..$^{5-7}$ 




FIG. 1. Repair-capacity disease progression model for multiple sclerosis (MS). The rapidly fluctuating disease activity reflected in short bursts of contrast enhancement (gadolinium-enhancing lesions) and transient lesion burden is set against a slowly progressing decline in repair capacity, the exhaustion of which indicates the onset of the progressive phase, in which inflammatory activity remains subthreshold and gives way to predominantly degenerative constituents of disease activity. The short-term time signature of new lesion behavior (bottom row) is hypothesized to change as an indicator of repair potential, from lesions that almost completely recover in a preclinical and relapsing phase to lesions in the progressive phase, which are permanent from the onset.

Both clinical characteristics and MRI findings point to a similar picture of slow but accelerated accrual of damage that eventually dissociates from baseline predictors, such as the load of gadolinium-enhancing lesions. Some of the most salient findings are discussed here.

\section{CLINICAL VARIABLES}

A comparison between early-onset and late-onset MS revealed overall more rapid progression initially in late- onset MS, which is in accord with a putative relapsing phase that remained subclinical (FIG. 1). The same comparison also revealed the rate of early progression (defined as time to Expanded Disability Status Scale EDSS score of 3) to be largely predictive of later progression (time to EDSS score of 6), but equally so for early and late onset, suggesting that once the disease has progressed beyond a particular point, a disease course is set, irrespective of time of onset. ${ }^{8}$

A similar pattern emerges for other predictive vari- 
ables. Baseline MRI for patients with an initial attack (i.e., clinically isolated syndrome, or CIS) is predictive of conversion to clinically definite MS, ${ }^{9}$ long-term progression, ${ }^{10}$ and failure to recover from an initial demyelinating event. ${ }^{11}$ Similarly, frequency and duration of early relapses and time to second episode ${ }^{12}$ all have predictive quality for future progression. ${ }^{11}$ However, such variables lose their predictive quality once an EDSS score of 4 is reached, ${ }^{11}$ which seems to be a landmark from which progressive disability measured by EDSS appears to accelerate. ${ }^{13}$ Hence, overall disease activity (commonly assessed in terms of new lesion occurrence) and its rate of conversion to permanent damage are two principal yet distinct factors that in part determine the overall rate of progression. For purposes of this discussion, we denote the ratio between the two as repair capacity, or repair potential. For example, a repair capacity of $60 \%$ would mean that less than half of all new disease activity leads to irreversible tissue damage, and an exhaustion of repair capacity suggests that all disease activity translates into permanent damage - that is, repair subsides.

\section{MRI VARIABLES}

MRI variables point to a similar picture of dissociation between disease activity and the proportion thereof that translates into permanent damage as the disease progresses. The prominent MRI markers for transient and permanent disease activity are new lesion occurrence and atrophy rates, respectively, commonly represented by contrast-enhancing lesions and brain parenchymal volume loss. Both markers and their mutual relationship show distinct changes during progression-most apparently between subtypes of relapsing and progressive MS.

Single lesion evolution, for example, exhibited a fourfold higher risk of residual damage in SPMS, compared with RRMS, whereas benign disease showed consistently better lesion recovery toward isointensity. ${ }^{14} \mathrm{~T}_{1}$ hypointense lesions (considered a hallmark of permanent matrix destruction) were more commonly observed in SPMS than RRMS,${ }^{15}$ and more new lesions remained $\mathrm{T}_{1}$ hypointense in SPMS. ${ }^{14,16}$ One of the few long-term studies involving MRI variables noted that MRI lesions in the first 2 years were associated with clinical outcome 13 years later, but not at the 2-year mark. ${ }^{17}$ Equivalent to the repair capacity hypothesis proffered here, this hysteresis was explained by a threshold effect beyond which pathology must progress before accrual of disability becomes manifest.

To what extent are the observed dissociations truly linked to a biological switch in the nature of the pathological process? Alternatively, how heavily are they influenced by varying sensitivity of measurement, both clinical and structural? For example, new enhancing lesions are commonly visible on MRI for less than 4 weeks, making a measure of the rate of lesion accrual dependent on both the interval spacing of follow-up and the overall rate of activity. ${ }^{18}$ Sampling error at a low activity rate will thus be significantly greater than at a high activity rate. Such differences in sensitivity for both clinical and MRI assessment may contribute to or mask actual changes between early relapsing and late progressive stages. For example, a disease subtype comparison of brain atrophy found no differences in global atrophy rates (brain parenchymal fraction), but significantly larger increases in central atrophy (ventricular fraction) for SPMS. ${ }^{19}$

The situation is similar for lesion burden. New lesion activity is transient and not equally apparent at all times, and not all focal activity reaches the threshold of MRI sensitivity. Such a view is supported by the finding that the variance of enhancing lesion counts over 6 months proved to be a better predictor for relapse rate than the actual baseline lesion number ${ }^{20}$-indicative not only of the temporal variability of disease activity, but also of the narrow time window for capturing contrast enhancement.

In a multivariate study of MRI predictors for clinical progression, including $\mathrm{T}_{1}$ and $\mathrm{T}_{2}$ lesions, white matter and gray matter atrophy, and magnetization transfer (MT) MRI, the MT was the best predictor. ${ }^{21}$ Although subject to short-term fluctuations similar to lesion contrast, MT has been strongly associated with demyelination and axonal loss in histology. ${ }^{22,23}$ MT is therefore expected to be more sensitive to the destructive aspects of disease activity, supporting the concept of subacute disease that reaches neither a clinical threshold nor becomes visible as focal MRI changes.

Assessment of disease progression in MS from MRI follow-up commonly involves one of three principal MRI variables: the number of new contrast-enhancing lesions, the volume of global $\mathrm{T}_{2}$ lesion burden, and an assessment of brain bulk, such as brain parenchymal fraction. ${ }^{24,25}$ Within this spectrum of measures, there are basically two avenues to obtain a measure of disease activity: 1) a marker directly linked (specific) to a particular physiological or pathological process, 2) a differential of disease severity measures, obtained at two or more different timepoints. An example of the first is the occurrence of gadolinium-diethylenetriamine penta-acetic acid (Gd-DTPA) contrast enhancement, capturing active focal blood-brain barrier patency, and an example of the second is rates of change of brain parenchymal fraction.

In the repair capacity model, the advanced MRI assessment strives to stage progression early, before atrophy or disability develop, by virtue of a sensitive and reliable measure of repair potential. In terms of repair, in vivo assessment must take into account that disease activity does not translate to disease severity in a linear fashion. Consequently, an early and specific MRI marker that distinguishes destructive from nondestructive activ- 


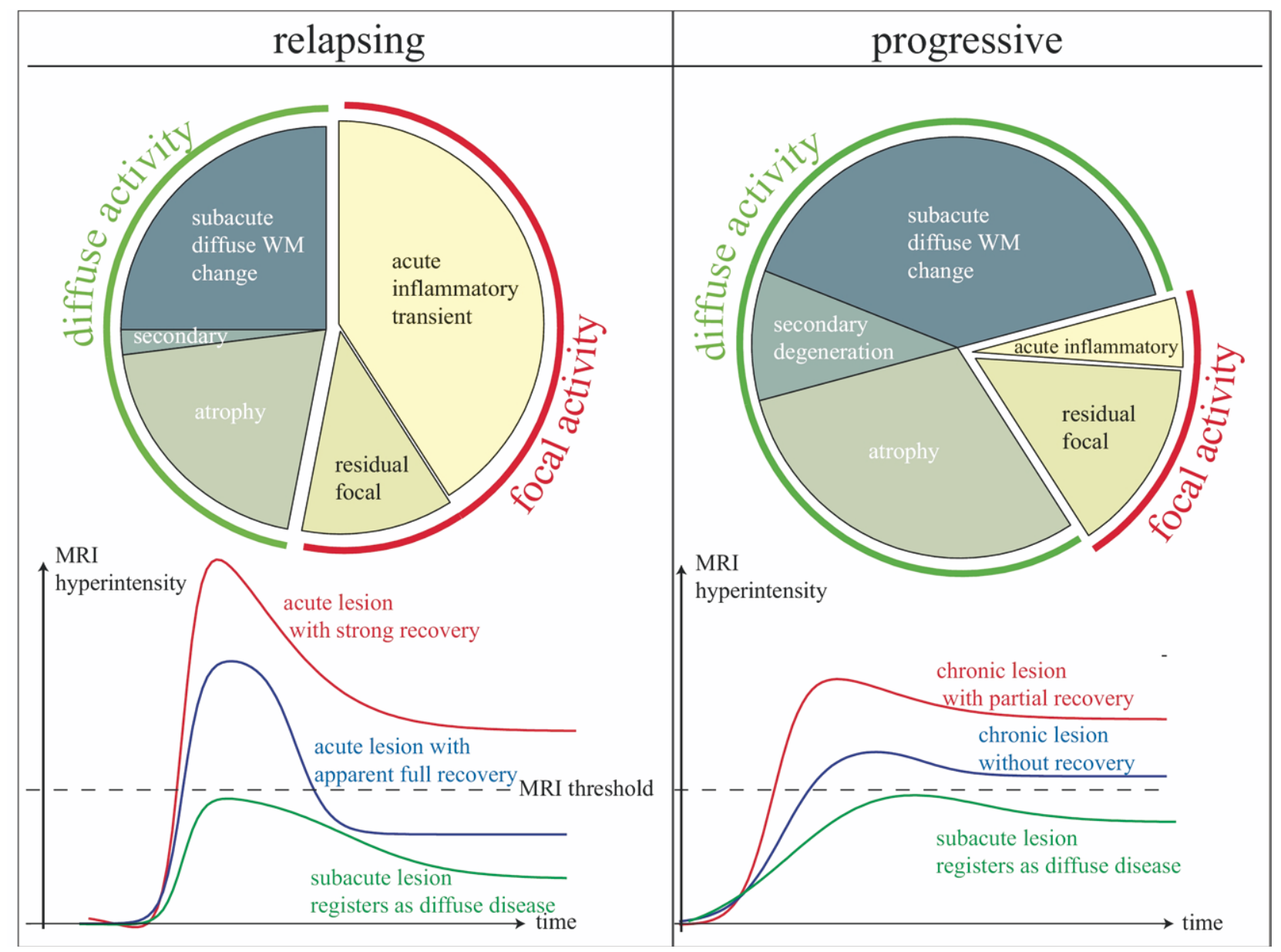

FIG. 2. Disease progression can be expressed as a shift in the proportions of visible disease activity and translations into permanent damage. Top: Distinguishing in relapsing and progressive MS between focal activity, visible as new MRI lesions, and diffuse activity, which registers as normal-appearing white matter (WM) change or atrophy. Many studies observe shifts in the constituent components; characteristic for relapsing MS is a strong level of transient inflammatory activity, with relatively little remaining as focal damage. Hallmarks of progressive MS are lower overall focal inflammatory activity with proportionally higher residual, and accrual of more diffuse damage. Bottom: The temporal profiles illustrate a corresponding shift in short-time lesion behavior. In a progressive stage, the transient response coinciding with active inflammation is reduced, and new lesions register directly as chronic damage. Subacute lesion activity below the MRI threshold will register only as diffuse disease and changes in normal-appearing WM. This corresponds to lesion time signatures in Figure 1.

ity is needed-that is, a dissociating metric for the component in MRI-visible disease activity that does contribute to permanent damage versus the component that does not.

Most of these predictors are related to inflammatory lesion activity, which can be associated with putative repair. Hence, to measure focal and transient disease activity to some extent also measures repair (FIG. 2). We may even argue that this dissociation between inflammation and degeneration is one aspect of repair. The stronger the dissociation, the more repair capacity is present to enable a response. In the transition from relapsing to progressive disease, blood-brain barrier patency, associated with bursts of inflammation, may give way to a reduced albeit chronic leakage with inflammatory activity that largely remains subacute (FIG. 2). This disease activity, however, translates directly into diffuse degenera- tion and eventually registers as irreversible damage in the form of atrophy. In such a model, a new acute MRI lesion becomes the visible indicator of inflammation elevating the response above the threshold of clinical and MRI sensitivity (green versus blue and red curves in FIG. 2).

That lesions below the MRI radar exist was revealed by a recent study comparing lesion conspicuity in highfield MRI ( $4 \mathrm{~T}$ versus $1.5 \mathrm{~T}$ ), which showed $\sim 50 \%$ increases in both detected lesion number and volume. ${ }^{26}$ Similarly, the MRI sensitivity for inflammation via contrast enhancement is strongly affected by acquisition protocol and contrast dose: triple-dose contrast showed substantially higher sensitivity than the single dose, ${ }^{27}$ and revealed the presence of significant inflammatory activity in early primary progressive disease. ${ }^{28}$

In summary, the MRI aspects of the repair-capacity model are as follows: 
- MS disease activity is visible on nonenhanced MRI as focal white matter lesions that appear and then, to a variable extent, disappear. To what extent this short-term MRI behavior is reflective of repair and long-term disease progression is still largely unknown.

- Not all MRI-visible disease activity leaves permanent tissue damage. That is, there are two aspects of MS disease activity: the overall visible MRI activity and the proportion that translates into permanent damage.

- MRI markers for repair in MS can come from either imaging with direct specificity toward repair processes, or from differential measures that indirectly reflect changing morphology.

- New lesion activity and permanent tissue destruction form two related but temporally disjointed processes, and we can model repair capacity as the level of that dissociation. That is, the more direct the translation of focal and transient activity into permanent damage, the lower the residual repair potential. At the two ends of the spectrum we have high repair capacity, where all or most activity remains subclinical, and exhausted repair capacity, marking the onset of secondary progressive stage, when all activity translates into permanent damage.

- Individual differences in this repair potential could lead to extended periods of subclinical disease, and could also explain the wide spectrum of disability, progression rates, and therapeutic effect.

\section{$T_{2}$ LESIONS REVISITED}

We will next review the $T_{2}$ lesion, particularly its short-term behavior, as a candidate marker of repair capacity. $\mathrm{T}_{2}$ MRI is an attractive candidate for such a marker, due to its prevalent use and availability of longterm follow-up data.

\section{Global lesion burden}

Longitudinal studies of clinical disease phenotypes or therapy efficacy commonly use changes in global $T_{2}$ lesion burden as an outcome measure. ${ }^{29}$ Large-scale clinical trials and cross-sectional studies generally use global $\mathrm{T}_{2}$ lesion volume as surrogate for disease severity and long-term trends thereof as indicators of disease activity and therapeutic effect. ${ }^{30-34}$ However, a pronounced short-term variability of $T_{2}$ lesion appearance, on the order of weeks and months, is known to be a substantial confounder of such global lesion burden assessment. Even though this waxing and waning characteristic of $T_{2}$ lesions has been known for a long time, and was first reported almost two decades ago, ${ }^{35,36}$ detailed characterizations of $\mathrm{T}_{2}$-weighted signal fluctuation are still sparse.
Difference image analysis showed that net change of global $\mathrm{T}_{2}$ lesion burden can underestimate actual new $\mathrm{T}_{2}$-weighted lesion volume change threefold. ${ }^{37}$ This suggests that substantially biased outcome measures may result from neglecting to differentiate the proportion of stable from new and potentially transient lesion burden in surrogates of either disease severity or activity. In its current form, consequently, global $\mathrm{T}_{2}$ lesion burden appears to be more specific as a diagnostic indicator ${ }^{38}$ than as a measure of disease severity. ${ }^{29}$

\section{$\mathrm{T}_{2}$ lesion evolution}

Lesion evolution is likely not only patient-specific, ${ }^{14}$ but also a phenomenon related to the disease stage. That is, lesion evolution patterns are good candidates for markers that can stage disease progression and subtype. The emphasis on evolution is important. In the mentioned theory of repair capacity, relations between disease activity and progression are more apparent from differential rather than absolute morphometry. For example, changes in enhancing lesion number and $\mathrm{T}_{2}$ lesion volume correlate with clinical activity (i.e., attacks) ${ }^{39}$ and clinical progression, ${ }^{37}$ as well as with markers of immunologic activity. ${ }^{40}$ Different levels not only of hypointensity but also of the rate of temporal change were observed when comparing remyelinating and inactive demyelinating $\mathrm{T}_{1}$ lesions. ${ }^{41}$

The need for more accurate assessment is enough to warrant a closer look at the dynamics of new lesion formation in the context of the underlying pathological processes of inflammation, degeneration, and repairespecially in the context of finding early markers of progression. The goal is to assess whether valuable information about the level and nature of repair processes may be encoded in how new lesions behave in the first few months after appearance (FIG. 2). The combination of fairly rapid changes and a probabilistic occurrence of new $\mathrm{T}_{2}$ lesions make such studies logistically difficult, particularly considering that today's therapeutic options can considerably reduce accrual of new lesions. Nevertheless, the potential benefit of obtaining early predictors of progression makes some form of differential measure from frequent MRI worthwhile.

MS lesion formation is generally divided into two MRI-visible phases: an acute phase, characterized by contrast enhancement, denoting blood-brain barrier patency, and a subacute or chronic phase characterized by $\mathrm{T}_{1^{-}}$and $\mathrm{T}_{2}$-weighted MRI signal abnormalities in the absence of MRI-visible blood-brain barrier leakage.

Serial studies of contrast enhancement showed a very brief window of observation, $<3$ weeks on average, ${ }^{18}$ and a duration-related likelihood for forming chronic hypointense lesions (chronic black holes). ${ }^{42}$ Although an increase of inflammatory activity reliably translates into an increase in chronic damage, a dissociation of $50 \%$ was 
observed where black hole formation occurred independently of inflammatory activity, ${ }^{42}$ equivalent to the chronic lesion type shown in Figure 2.

\section{$T_{2}$ lesion intensity dynamics: time-series analysis}

Using methods of time-series analysis, an alternative approach to the morphometry of $\mathrm{T}_{2}$ lesion evolution was presented by extracting the dynamics of change from pixel-wise temporal intensity variations directly, rather than from regressions or models of lesion volume or shape. ${ }^{43}$ One of the principal motivations for this approach is that of enhanced sensitivity to change (our metric of interest), in that this approach circumvents the data reduction associated with integrating or thresholding the pixel data into a single scalar variable, as necessary for purely volumetric variables. An alternative to pure lesion volume is, for example, the weighted hyperintensity metric shown in Figure 3, which sums all regional pixels, weighted by their relative hyperintensity above baseline.

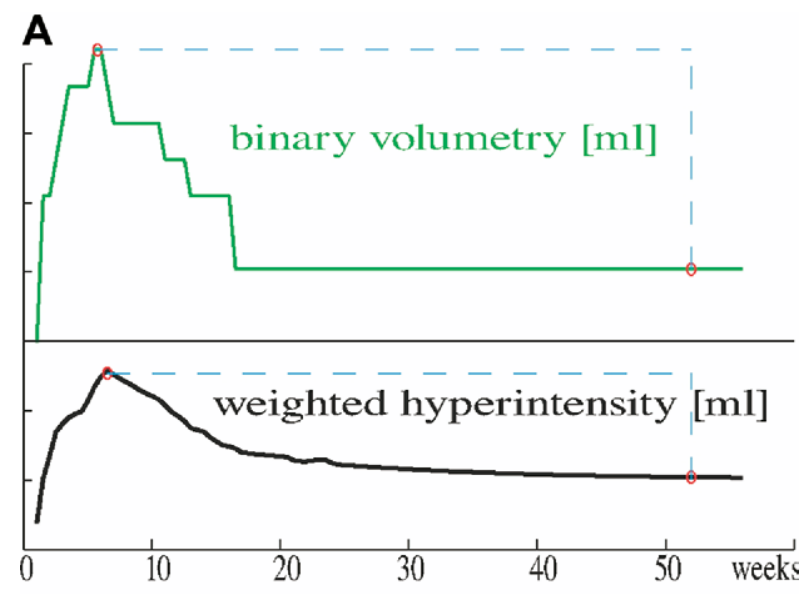

B Binary Volumetry $=\sum$ threshold pixel count $/ \sum$ all pixels

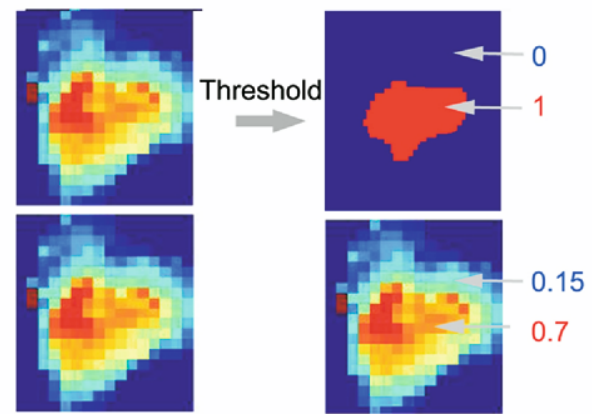

Weighted Volumetry $=\Sigma$ all pixels $\cdot$ relative hyperintensity

FIG. 3. A, Lesion volumetry (green) vs. weighted hyperintensity (black), shown as temporal profile from multiple exams over 1 year: volumetry involves a binary measure that must decide whether an image pixel is considered hyperintense or not. This decision leads to a substantially cruder representation of the temporal change, apparent from the discontinuities in the profile shape. B, A weighted hyperintensity measure circumvents the binary decision by weighing each voxel count by its relative hyperintensity.
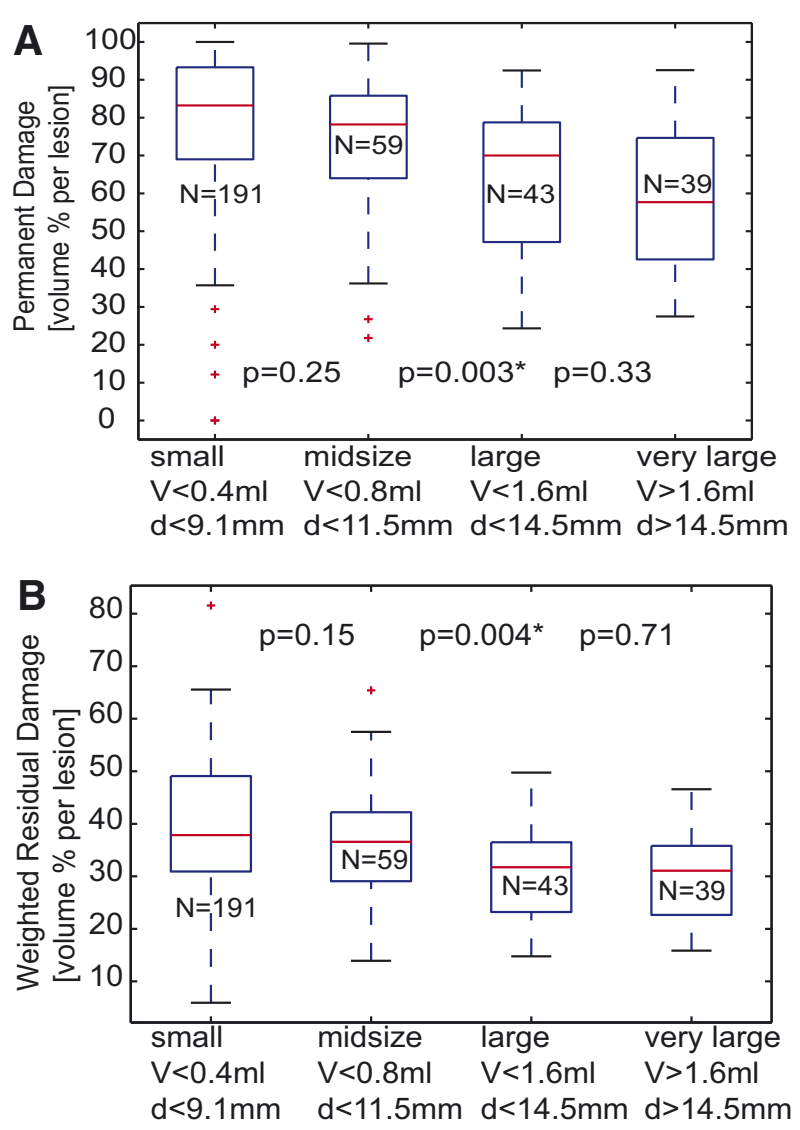

FIG. 4. A, Proportions of permanent lesion volume a defined as pixels with $\geq 20 \%$ residual hyperintensity. The proportion of permanent lesion volume decreases for greater lesion size $(P=$ 0.003 ). B, An alternative and potentially more accurate measure of weighted residual damage is the integrated residual water (a weighted sum of pixel hyperintensity). The same size dependence emerges. Abbreviations: d, diameter; N, group size (number of lesions); $p$, significance of group comparison to the next smaller size; V, volume;

In a classic atrophy study or paradigm, tissue contrast changes gradually over time until it falls below a threshold and is no longer counted as part of the tissue class and hence is classified as atrophied. This gradual change is not captured if the study outcome measure involves volumetric variables only. In binary volumetry, not only is the sensitivity to change reduced by the thresholding, but also diffuse changes that do not result in structure shrinkage are not captured at all, because these manifest as global (structure-wide) intensity changes, not peripheral changes. The time-series analysis concept is complementary, in the sense that a mechanism is provided to detect and characterize those changes, possibly before they translate into irreversible tissue loss.

\section{$\mathbf{T}_{2}$ lesion recovery as a marker for repair potential?}

We suggest short-term recovery of new T2 lesions as a marker for assessing repair potential in MS. Repair potential itself is hypothesized as a marker for disease progression. In the time-series analysis paradigm, we 
denote recovery as recovery of the white matter signal toward isointensity. That is, the proportion of new lesion signal recovery is hypothesized to relate to the patient's current repair potential.

We then arrive at the following hypothesis: Patients with greater levels of short-term lesion recovery (less residual lesion hyperintensity) possess greater repair potential and express slower disease progression.

If this is true, then long-term follow-up should reveal lower brain atrophy rates as well as lower accrual of diffuse disease, matrix destruction (chronic black holes), and ultimately lower disability. For a measure of lesion recovery, we seek to quantify the transient versus permanent damage on a per-lesion or even per-pixel basis. An example will illustrate the importance of sensitivity in measures of change. A volumetry approach requires a threshold for residual hyperintensity. That is, we have to first define what constitutes permanent damage and then count the image pixels matching those criteria. We compare such a count with a weighted measure in Figure 3.

Figure 4 shows examples of residual (nontransient) lesion burden for a cutoff at $20 \%$ (i.e., a pixel with residual hyperintensity of $>20 \%$ above baseline is considered permanent damage). The average proportions of nontransient lesion volume in this case are $74 \% \pm 21 \%$ of a lesion (relative to maximum size). The comparison examines effects of lesion size: larger lesions expressed significantly smaller proportions of permanent lesion volume, both for standard volumetry and for hyperintensity-weighted volumetry. The latter, as we have noted, is a potentially more practical measure without the need for an intensity threshold. It represents a weighted sum of relative hyperintensities over all lesion pixels, representing an estimate of total residual water or total residual damage. The same relation to lesion size is apparent, with an average residual of $36 \% \pm 12 \%$ (FIG. $4 \mathrm{~b}$ ). Note also that the variability of the measure is less and more evenly spread, providing enhanced sensitivity.

To place $T_{2}$ lesion activity in the context of a disease progression model, the following are key questions about the temporal characteristics of new $T_{2}$ lesion activity: How long is a new $\mathrm{T}_{2}$ lesion active? How much of this $\mathrm{T}_{2}$ activity is active inflammation? How much $\mathrm{T}_{2}$ activity is transient? Does lesion size matter? In the following sections we briefly address each of these questions by reviewing recent findings from a natural-history (untreated) MS cohort followed with high-frequency MRI ${ }^{39}$ and analyzed with time-series analysis methods ${ }^{43}$ and time-series modeling. ${ }^{44}$

\section{How much $T_{2}$ activity is transient?}

Pixel-wise time-series analysis of MRI intensity dynamics and modeling showed that the dynamics of new MS lesions are spatially very heterogeneous: the bound- ary of a new lesion behaves very differently from the center. ${ }^{44}$ Examples of maps of lesion behavior typical for RRMS are shown in Figure 5. Consistent concentric characteristics are apparent (especially for larger lesions) for both peak hyperintensity and residual. A positive relation between the level of peak intensity and amount of residual damage is also qualitatively apparent. That is, a lower level of peak intensity appears to be predictive of the chance of recovery.

Comparison of the hyperintensity and the residual map also shows the spatial extent to which a lesion recovers and where. This heterogeneity illustrates that representing a lesion or even global lesion burden with a single number is problematic. White matter lesions represent four-dimensional structures in space and time, and an accurate description of their temporal behavior requires careful definition in both the spatial and intensity domain. The amount of transient signal change is therefore best described by empirical cumulative distribution functions (Kaplan-Meier), as shown in Figure 6.

\section{How long is a new $T_{2}$ lesion active?}

New $T_{2}$ lesions in a natural-history (i.e., untreated) cohort exhibited an active phase of 3-4 months. ${ }^{44}$ After this phase, no significant resolving or change toward isointensity of lesions in a long-term scope of 3-4 years was observed. This 3-month activity also determines our window of opportunity for observing $T_{2}$ change. That is, follow-up MRI intervals of $>3$ months will likely not yield reliable or useful measurements of new lesion dynamics. On the other hand, follow-up MRI at frequencies of $<1$ month is currently impractical and therefore would have limited application in a clinical setting.

\section{How much $T_{2}$ activity is active inflammation?}

A separate study on the duration and evolution of contrast enhancing lesions found substantial heterogeneity in duration, and associations between early lesion growth and the overall duration of enhancement. ${ }^{18}$ The duration itself has been shown to associate with the likelihood of the lesion becoming a chronic black hole ${ }^{42}$; hence, an association between the initial lesion behavior and the level of residual damage appears feasible.

We found the duration of active contrast enhancement to be $\sim 1-3$ weeks, ${ }^{18}$ significantly shorter than the $2-3$ month activity observable on $\mathrm{T}_{2}$ (FIG. 7). $\mathrm{T}_{2}$ activity continues long after contrast enhancement subsides, defining a subacute phase of lesion activity and demonstrating sensitivity to processes other than inflammation and edema reabsorption. ${ }^{44}$ Such sensitivity adds further support to the use of $T_{2}$ recovery as a marker for overall repair capacity and finally progression. (Pathophysiological and etiological interpretations of this subacute phase are discussed separately.) 


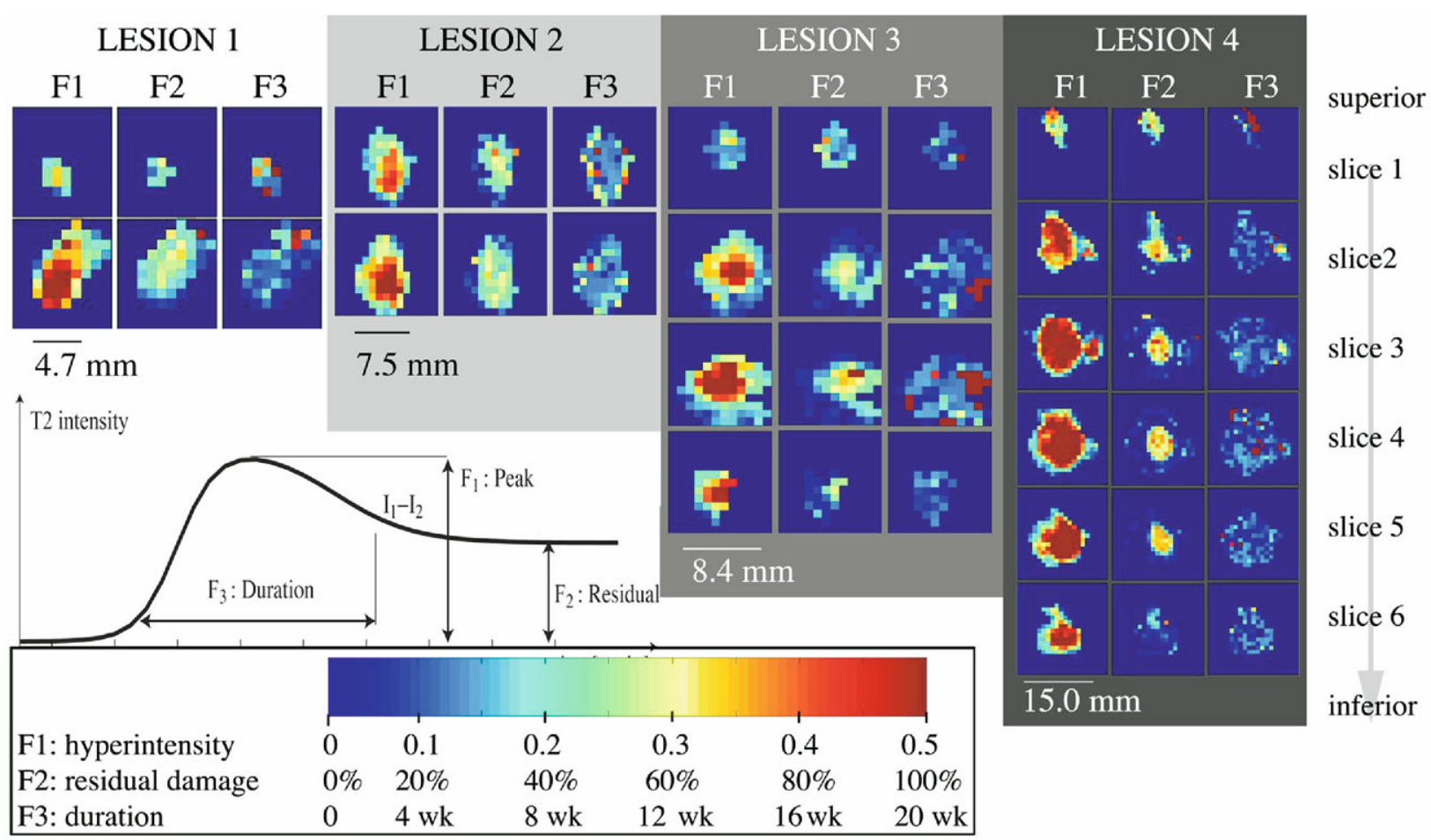

FIG. 5. An example with maps of four lesions of different size. Column triplets for each lesion show hyperintensity fraction above baseline (F1), residual damage in \% (F2), and duration in weeks (F3). Rows show all slices of the lesion (slice thickness: 3 mm). Lesions are shown at individual scales (separate scale bar for each lesion). Concentric patterns and a correlation between higher levels of intensity and greater residual are apparent. Residual damage tends occur in areas of greatest hyperintensity. Note also how the proportions of residual damage (F2) are lower for larger lesions.

\section{Does lesion size matter?}

A comparison of the small and large lesion depicted in Figure 5 shows that the two sizes behave very differently. The proportion of residual hyperintensity is substantially smaller in the larger lesion. The same disproportional recovery was observed consistently across subjects (FIG. 6). Such a trend toward smaller proportions of permanent damage in larger lesions suggests that a case with many small lesions could represent worse (i.e., more permanent) damage than a case with equivalent total lesion burden comprised of a few large lesions.

Methodologically, this trend also indicates that the level to which single-timepoint lesion burden measures are confounded by transient (nondamaging or minimally damaging) activity depends on lesion size. In other words, the volume of lesion hyperintensity does not

a

b
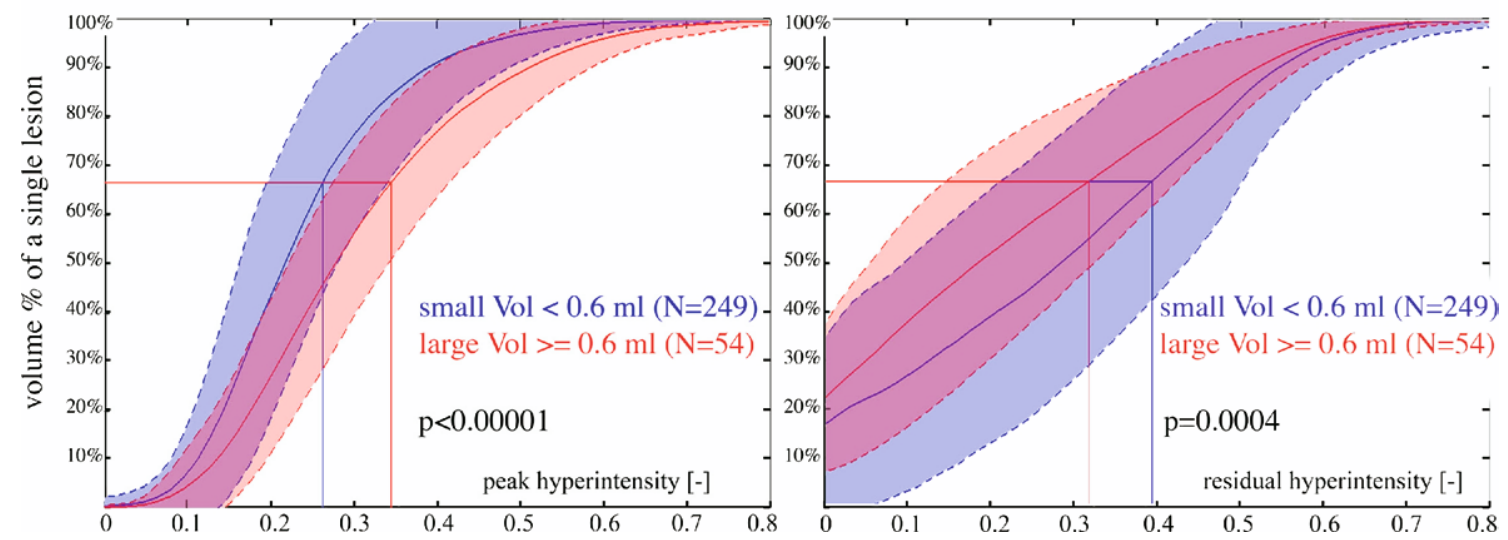

FIG. 6. Lesion property distributions (Kaplan-Meier, estimated cumulative distributions), stratified by lesion size, with blue and red bands denoting small and large lesions, respectively. The center curve and colored band give the mean \pm standard deviation across all lesions, respectively. The vertical line indicates a $2 / 3$ cutoff. For example, two thirds of a small lesion retains a hyperintensity of $\leq 0.4$ (blue line in panel b), which drops for large lesions. Larger lesions (red) appear to have different hyperintensity distributions (a) and proportionally less residual hyperintensity (b). 




FIG. 7. An example comparing new $T_{2}$ lesion activity (bottom row) with contrast enhancement $\left[T_{1}\right.$-Gd-DTPA, top row]. A new enhancing lesion appears at week 5 (yellow number marks follow-up in weeks). Note that active enhancement, indicating blood-brain barrier breakdown, dissipates after 4-5 weeks, whereas transient $T_{2}$ activity continues for 10-15 weeks.

translate linearly into a metric for disease burden: twice the lesion load is not twice the damage.

The heterogeneity of the dynamic response is captured better by distributions (FIG. 6) than by an integrated measure. Such curves are not easy to interpret, however. Figure 8 shows examples of different lesion fates, illustrating the numbers and curves reported here. Three distinct lesion fates are illustrated, with recovery proportions ranging from $18 \%$ to $83 \%$. The single distribution curves in Figure 8 show more clearly than do the averages in Figure 7 that transient and permanent lesions also differ in their spatial pattern. The relatively large haloshaped proportion of recovering hyperintensity (possibly originating from resolving edema) is largely absent in the chronic permanent lesion.

\section{Does $\mathbf{T}_{\mathbf{2}}$ activity affect or predict progression?}

Do these patterns of evolution and activity agree with a progression theory of an exhausted repair potential? In other words, is there predictive value in lesion recovery as an early MRI surrogate of disease progression as measured by other variables, such as brain parenchymal fraction or EDSS score?

Clues to the specificity of new lesion formation as a progression marker arise from direct comparison of le- sion behavior with established measures of progression or from comparisons among patients in a relapsing versus progressive phase. An example of two new lesions from two MS patients is shown in Figure 9. The lesion in subject 1 is substantially larger, but also expresses profound recovery, whereas the smaller lesion of subject 2 retains most of its peak hyperintensity. Our model would thus associate greater repair capacity with subject 1 and predict slower progression. Comparison of brain parenchymal fraction and EDSS progression shows indeed that the smaller lesion was associated with substantially faster progression in terms of both tissue loss and accrual of disability.

Another example of lesion evolution in relapsing versus progressive MS is shown in Figure 10. The new lesion in the progressive stage exhibits less inflammation, smaller size, and minimal recovery after its initial appearance, compared to a lesion in RRMS, which shows stronger enhancement but also comprehensive recovery.

Thus, the lesion time signatures appear to be in accord with a theory of a repair potential that is diminished over time and is associated with inflammatory activity or the MRI visibility thereof. We may



FIG. 8. New $T_{2}$ lesions vary substantially in their evolution patterns: (a) a case of almost complete recovery (83\% transient volume), (b) small focal residual (73\% transient volume), (c) a lesion with little recovery and a large residual (18\% transient volume). The images show timepoints of one slice of the lesion (numbers indicate weeks of follow-up). The color maps F1, F2, and F3 represent hyperintensity, residual, and duration, respectively (as in FIG. 5). The graph on the right shows the Kaplan-Meier distribution for each lesion, showing how much of each lesion retains what level of hyperintensity. For example, for the lesion in (c), $\sim 18 \%$ of lesion pixels had residual hyperintensity of $\leq 20 \%$. 
A
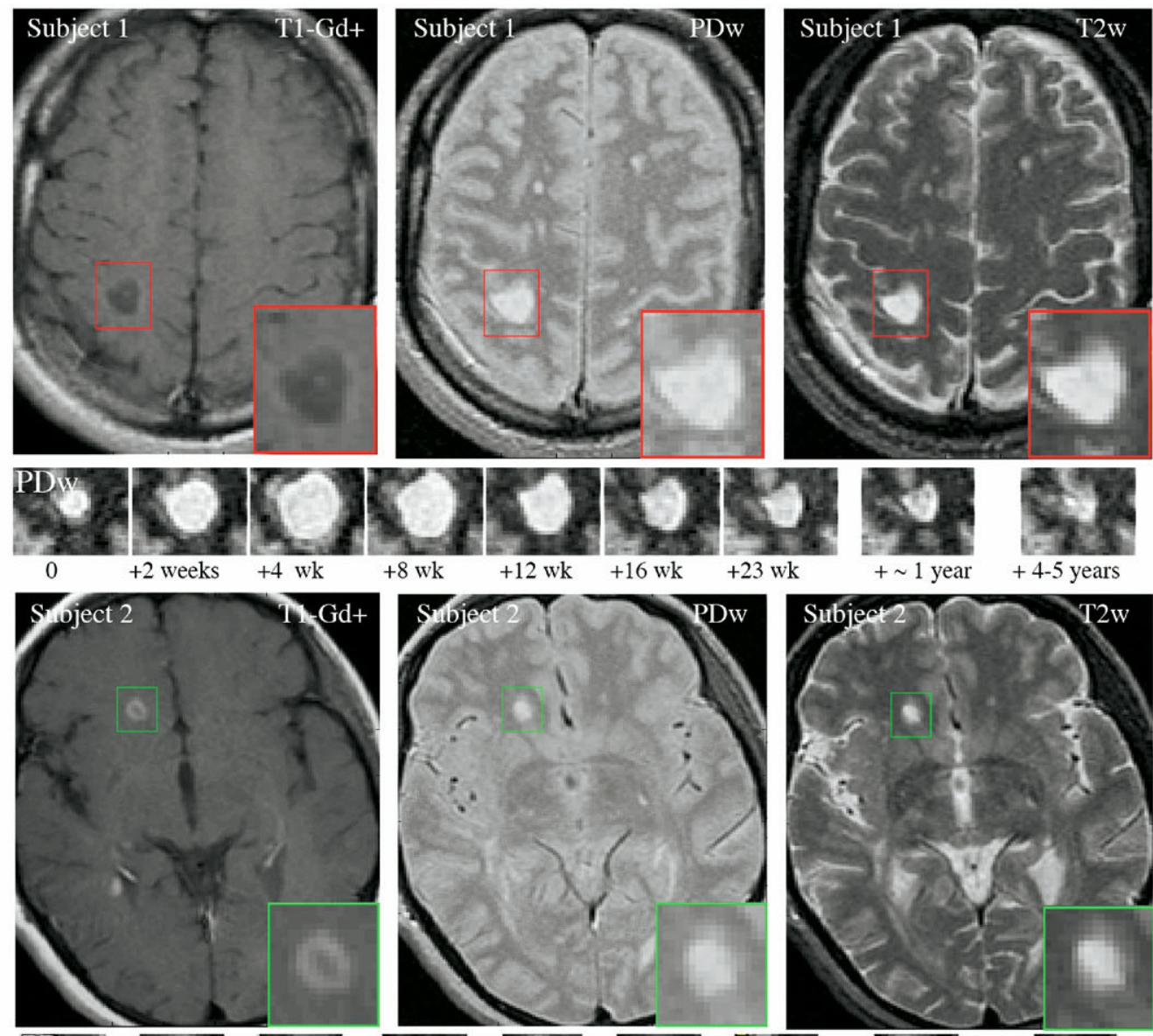

$23 \mathrm{wk}$

$+\sim 1$ year

$+4-5$ years
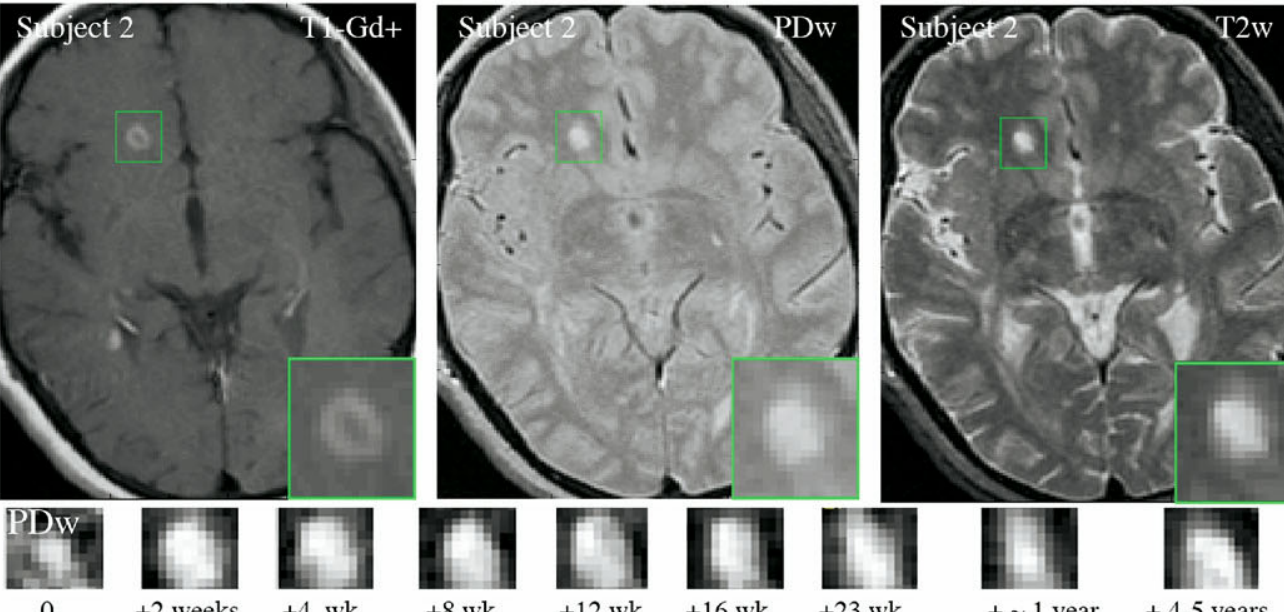

B
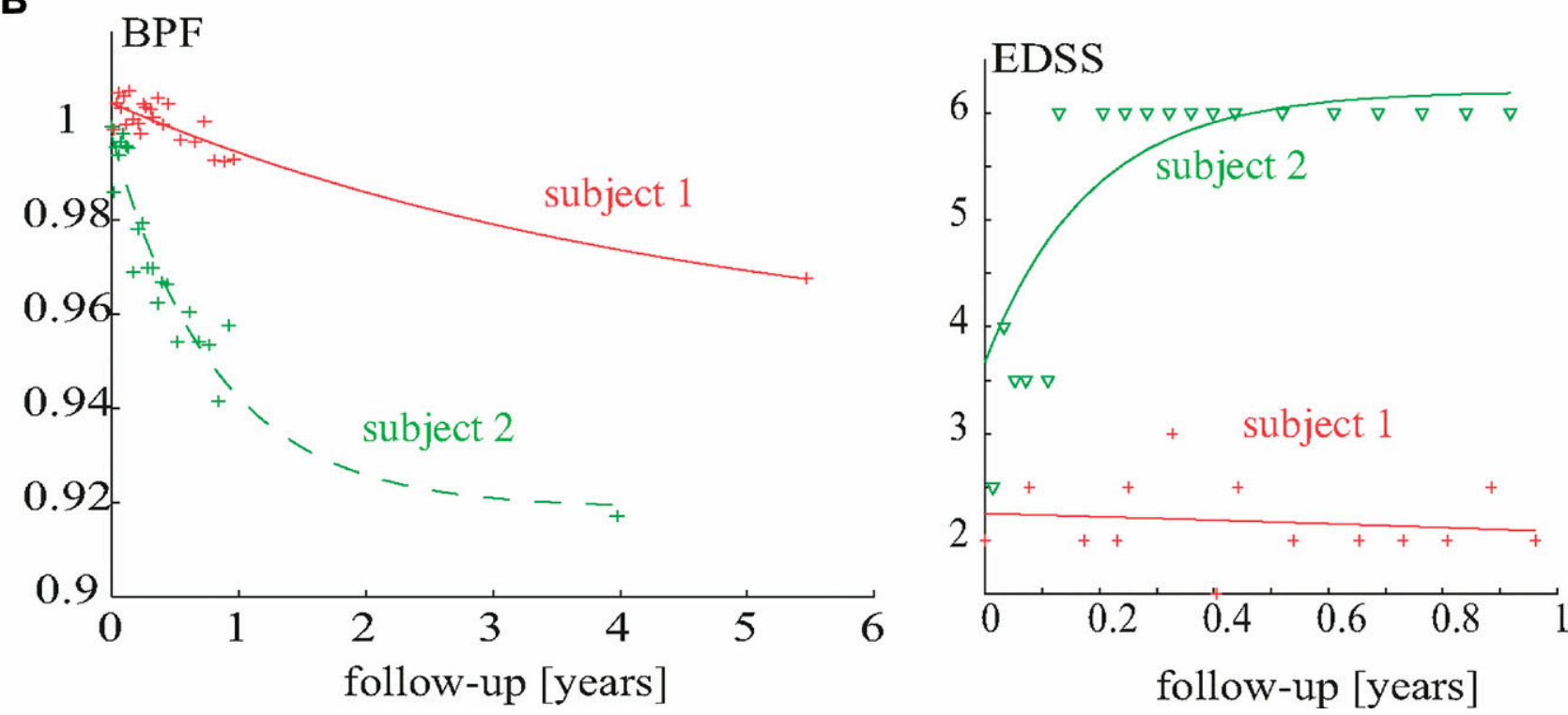

FIG. 9. The clinical impact: A, A comparison, for two multiple sclerosis (MS) patients, of a short-term lesion evolution and B, long-term progression in terms of disability [Expanded Disability Status Scale (EDSS) score] and atrophy (brain parenchymal fraction, or BPF). In A, columns (left to right) show $\mathrm{T}_{1}$-gadolinium-enhancing (T1-Gd+), proton density-weighted (PDw), and $\mathrm{T}_{2}$-weighted (T2W) MRI. The evolution for these 2 lesions is shown beneath (from the PDw series). Note the greater recovery of lesion 1 (red outline), despite the lesion being larger than for lesion 2 (green outline). Subject 1 exhibited substantially lower disability than subject 2. 


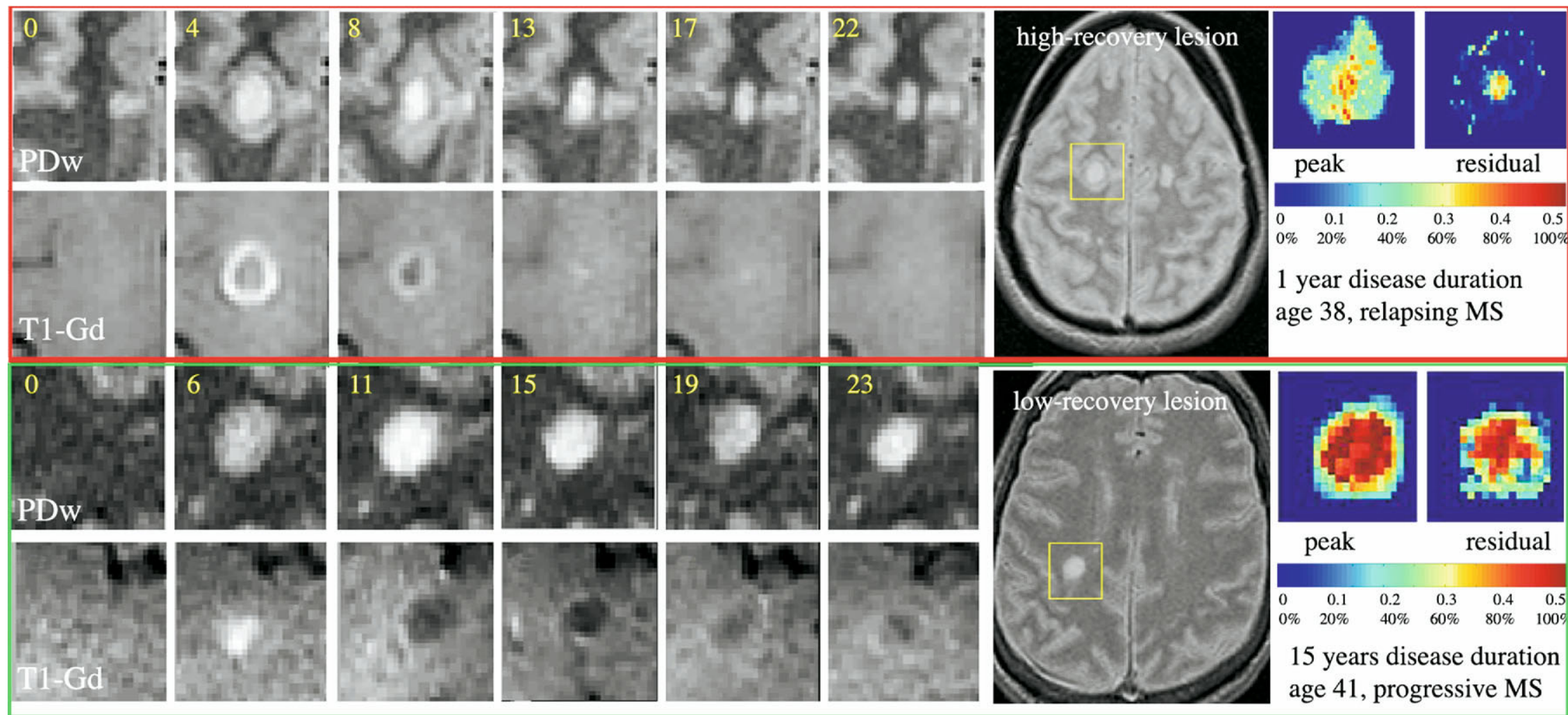

FIG. 10. Lesion history comparison in relapsing versus progressive MS. Lesion 1 (top: red outline) appeared in an MS patient within the first year of diagnosis, showing strong inflammatory activity (ring enhancement, $\mathrm{T}_{1}$-Gd), but also comprehensive recovery. Lesion 2 (bottom: green outline) appeared in an MS patient with 11 years of disease duration (15 years after the first symptom): enhancement ( $\left.T_{1}-G d\right)$ is short and followed by persistent hypointensity on $T_{1}$. The $T_{2}$ lesion is small and shows only little recovery in size or hyperintensity. Numbers in yellow indicate follow-up time in weeks. The Expanded Disability Status Scale (EDSS) score at the time of scan was 1.5 and 6 for patients 1 and 2 , respectively. 
attribute the latter (i.e., the level of visible lesion activity) to the level of responsiveness of the parenchymal repair mechanism. Translated into a crosssectional scenario, this would match exactly the observation that the variability of new lesion appearance was a stronger predictor of disease activity than was the overall number of lesions. ${ }^{20}$ Note that the level and dynamic activity of a single new lesion in this paradigm is not to be mistaken for the overall level of disease activity, which is most commonly assessed as the total number of new lesions observed.

\section{DISCUSSION: PATHOPHYSIOLOGICAL AND ETIOLOGICAL INTERPRETATIONS}

Discussion of the role of inflammation in MS was rekindled by histopathological records of distinct lesion patterns that dissociate from the classic picture of T-cellmediated tissue damage, particularly lesions in which remyelination is not observed but oligodendrocyte loss is apparent. ${ }^{45,46}$

Many reported histological patterns are reflective of different evolution stages: from acute active plaques with abundant macrophages and myelin breakdown, to active (smoldering) rim lesions with macrophage presence concentrated at the rim, to inactive plaques without myelin breakdown. ${ }^{4}$ Recent studies also showed remyelination to be more extensive than previously assumed, ${ }^{47}$ giving new impetus to the search for an in vivo marker of repair and calling for new paraclinical markers of how tissue destruction occurs in vivo. ${ }^{48}$ The residual heterogeneity of lesion patterns observed in time-series analysis studies implies similar variability in progression and destructive nature of lesion formation. ${ }^{43,44}$

A previous analysis of the evolution of contrast-enhancing (Gd-DTPA) lesions on $\mathrm{T}_{1}$-weighted MRI yielded an average duration of enhancement of 3 weeks. ${ }^{18}$ A side-by-side comparison of Gd activity with the $T_{2}$ activity shows correspondence with the first part of the transient or active portion of a $\mathrm{T}_{2}$-lesion (FIG. 7). After 4-5 weeks, active Gd enhancement has subsided and transient $T_{2}$ hyperintensity continues, supporting a model that marks active inflammation and water or edema reabsorption (and possibly some level of repair or gliosis) as the chief contributors to the visible $\mathrm{T}_{2}$ activity in the first 10 weeks of new lesion evolution. ${ }^{44}$

In that study, the duration of new $\mathrm{T}_{2}$ lesion activity was 10 weeks on average, with the spectrum reaching to $\sim 5-6$ months. ${ }^{44}$ The first few weeks of a new $\mathrm{T}_{2}$ lesion correspond with Gd-DTPA contrast enhancement, indicating active inflammation and edema development. Although this coincides with the strongest $T_{2}$ changes in both hyperintensity and lesion size (FIG. 7), the present results clearly indicate a subacute phase with significant $\mathrm{T}_{2}$ change after contrast enhancement has subsided. A separate analysis of a subgroup of the same patient cohort yielded average enhancement durations of 3 weeks, with $97 \%$ of lesions enhancing for less than 2 months. ${ }^{18}$

On average, $80 \%$ of a new lesion (measured at peak) resolves within the initial 10 -week period, which is most commonly interpreted as dominated by the reabsorption of inflammatory water. ${ }^{36}$ However, the extended range of the subacute phase spectrum of 5-6 months is also compatible with $T_{2}$ changes reflecting processes other than water reabsorption (e.g., remyelination and gliosis).

Because disease-modifying therapies aim to alter the pathophysiological chain of inflammation, demyelination and axonal loss, a therapeutic effect may be more readily apparent as a change in lesion dynamics, their recovery rate and level, rather than as a change in total lesion burden or enhancing lesion number. Emphasis on disease dynamics was also bolstered by recently proposed alternative views of neuroprotective aspects of autoimmune responses,${ }^{49}$ which stress the timing and extent of T-cell expression as pivotal in characterizing the disease.

Residual hyperintensity showed concentric patterns of lesion dynamics, with the most permanent damage in the lesion center (FIG. 5). Concentric patterns were also described in histopathologic studies, such as active plaque edges with accumulating macrophages. ${ }^{46}$ Thus, if the observed dynamic MRI patterns also relate to pathogenic differences, MRI surrogates of disease activity of greater specificity could be gained by differentiating lesion types by their level of hyperintensity and the temporal change thereof.

If we attribute the rapid return toward isointensity during the first 10 weeks to inflammatory water reabsorption, subsequent changes or trends toward isointensity could be attributed to repair processes (e.g., remyelination). Likewise, trends toward stable or greater hyperintensity could be markers of degeneration (e.g., further demyelination or axonal loss). In such a scenario, the difference in recovery level between 10 and 20 weeks could serve as a marker for the level of repair.

In this review, we have focused on $\mathrm{T}_{2}$-weighted signal dynamics, but the short-term behavior of $\mathrm{T}_{1}$ lesions is not radically different qualitatively; for example, $44 \%$ of initially hypointense $T_{1}$ lesions returned to isointensity. ${ }^{50}$ Short-term $T_{1}$ hypointensity changes of course are also readily attributable to edematous water reabsorption, but chronic $\mathrm{T}_{1}$ hypointensity (chronic $\mathrm{T}_{1}$ black holes) has repeatedly been suggested as a marker for permanent matrix destruction, and it may indeed contain viable complementary information. It would therefore be of great interest to determine if the mid- and long-term $T_{1}$ dynamics dissociate from the $T_{2}$ measures discussed here.

Treatment effects have been shown by assessing the number of new $\mathrm{T}_{2}$ lesions evolving into $\mathrm{T}_{1}$ black holes. ${ }^{51}$ An extension of lesion evolution assessment and their 
dynamics to multicontrast dynamics would therefore permit predictions across different MRI contrast mechanisms, possibly yielding much sought-after pathologically specific MRI surrogates of MS disease activity.

The disproportionately smaller residual for larger lesions expresses a nonlinear relationship between active lesion burden and residual damage, which, as outlined in Figure 1 , is likely subject to change as the disease progresses and repair capacity is becoming depleted. To the extent that peak lesion size implicates the inflammatory response, larger lesions become hallmarks of both stronger immune activity and greater repair-which is noteworthy, in light of recently extended positive aspects of inflammation in MS pathogenesis. ${ }^{49}$

Pathogenic interpretations become viable once we can rule out that the observed size effect stems from MRI resolution limitations and associated bias in sensitivity. Consequently, many small new lesions could indicate less repair and more destruction than a case with a few large new lesions of equivalent volume. Most of the reviewed per-lesion and per-patient analyses support the notion that the level and amount of new lesion activity may be good surrogates for disease activity, but not necessarily specific to how destructive this activity is.

\section{CONCLUSION}

We have discussed serial MRI morphometry, particularly the short-term behavior of new $\mathrm{T}_{2}$ lesions, in the context of a disease progression model that features a repair capacity that, when exhausted, marks the conversion or progression from relapsing to progressive disease. Both clinical and MRI variables are in support of such a model. The challenge is the search for an MRI surrogate that can stage progression earlier, before atrophy or disability develop.

Findings from serial MRI suggest that the short- and mid-term term behavior of new $\mathrm{T}_{2}$ lesions may have potential as such a marker: time signatures with lower rates of lesion recovery may represent lower repair and greater proximity to a progressive stage. Individuals with low transient lesion turnover appear to undergo more rapid progression and atrophy.

Because disease-modifying therapies aim to alter the pathophysiological chain of inflammation, demyelination, and axonal loss, a therapeutic effect may be more readily apparent as a change in lesion dynamics and in their recovery rate and level, rather than as a change in total lesion burden or number of enhancing lesions.

Acknowledgments: This work was supported in part by funding provided by the National Multiple Sclerosis Society (RG 3574-A-1) and the National Institutes of Health (R01 NS35142; P41 RR13218-01).

\section{REFERENCES}

1. Weinshenker BG, Bass B, Rice GP, et al. The natural history of multiple sclerosis: a geographically based study. I. Clinical course and disability. Brain 1989;112:133-146.

2. Runmarker B, Andersen O. Prognostic factors in a multiple sclerosis incidence cohort with twenty-five years of follow-up. Brain 1993;116:117-134

3. Kutzelnigg A, Lucchinetti CF, Stadelmann C, et al. Cortical demyelination and diffuse white matter injury in multiple sclerosis. Brain 2005;128:2705-2712.

4. Prineas JW, Kwon EE, Cho ES, et al. Immunopathology of secondary-progressive multiple sclerosis. Ann Neurol 2001;50:646657.

5. Filippi M, Rovaris M, Iannucci G, et al. Whole brain volume changes in patients with progressive MS treated with cladribine. Neurology 2000;55:1714-1718.

6. Molyneux PD, Kappos L, Polman C, et al. European Study Group on Interferon $\beta-1 \mathrm{~b}$ in Secondary Progressive Multiple Sclerosis. The effect of interferon $\beta$-1b treatment on MRI measures of cerebral atrophy in secondary progressive multiple sclerosis. Brain 2000;123:2256-2263.

7. Paolillo A, Coles AJ, Molyneux PD, et al. Quantitative MRI in patients with secondary progressive MS treated with monoclonal antibody Campath 1H. Neurology 1999;53:751-757.

8. Tremlett H, Devonshire V. Is late-onset multiple sclerosis associated with a worse outcome? Neurology 2006;67:954-959.

9. Tintore M, Rovira A, Rio J, et al. Baseline MRI predicts future attacks and disability in clinically isolated syndromes. Neurology 2006;67:968-972.

10. Chard DT, Brex PA, Ciccarelli O, et al. The longitudinal relation between brain lesion load and atrophy in multiple sclerosis: a 14 year follow up study. J Neurol Neurosurg Psychiatry 2003;74: $1551-1554$

11. Confavreux C, Vukusic S, Adeleine P. Early clinical predictors and progression of irreversible disability in multiple sclerosis: an amnesic process. Brain 2003;126:770-782.

12. Weinshenker BG, Bass B, Rice GP, et al. The natural history of multiple sclerosis: a geographically based study. 2. Predictive value of the early clinical course. Brain 1989;112:1419-1428.

13. Kappos L, Traboulsee A, Constantinescu C, et al. Long-term subcutaneous interferon $\beta$-1a therapy in patients with relapsing-remitting MS. Neurology 2006;67:944-953.

14. Minneboo A, Uitdehaag BM, Adèr HJ, et al. Patterns of enhancing lesion evolution in multiple sclerosis are uniform within patients. Neurology 2005;65:56-61.

15. Truyen L, van Waesberghe JH, van Walderveen MA, et al. Accumulation of hypointense lesions ("black holes") on T1 spin-echo MRI correlates with disease progression in multiple sclerosis. Neurology 1996;47:1469-1476.

16. van Waesberghe JH, van Walderveen MA, Castelijns JA, et al. Patterns of lesion development in multiple sclerosis: longitudinal observations with T1-weighted spin-echo and magnetization transfer MR. AJNR Am J Neuroradiol 1998;19:675-683.

17. Rudick RA, Lee JC, Simon J, Fisher E. Significance of T2 lesions in multiple sclerosis: a 13-year longitudinal study. Ann Neurol 2006;60:236-242.

18. Cotton F, Weiner HL, Jolesz FA, Guttmann CR. MRI contrast uptake in new lesions in relapsing-remitting MS followed at weekly intervals. Neurology 2003;60:640-646.

19. Kalkers NF, Ameziane N, Bot JC, et al. Longitudinal brain volume measurement in multiple sclerosis: rate of brain atrophy is independent of the disease subtype. Arch Neurol 2002;59:1572-1576.

20. Kappos L, Moeri D, Radue EW, et al. Gadolinium MRI Metaanalysis Group. Predictive value of gadolinium-enhanced magnetic resonance imaging for relapse rate and changes in disability or impairment in multiple sclerosis: a meta-analysis. Lancet 1999; 353:964-969.

21. Agosta F, Rovaris M, Pagani E, et al. Magnetization transfer MRI metrics predict the accumulation of disability 8 years later in patients with multiple sclerosis. Brain 2006;129:2620-2627.

22. Schmierer K, Scaravilli F, Altmann DR, et al. Magnetization trans- 
fer ratio and myelin in postmortem multiple sclerosis brain. Ann Neurol 2004;56:407-415.

23. van Waesberghe JH, Kamphorst W, De Groot CJ, et al. Axonal loss in multiple sclerosis lesions: magnetic resonance imaging insights into substrates of disability. Ann Neurol 1999;46:747-754.

24. Miller DH, Albert PS, Barkhof F, et al. US National MS Society Task Force. Guidelines for the use of magnetic resonance techniques in monitoring the treatment of multiple sclerosis. Ann Neurol 1996;39:6-16.

25. Filippi M, Horsfield MA, Adèr HJ, et al. Guidelines for using quantitative measures of brain magnetic resonance imaging abnormalities in monitoring the treatment of multiple sclerosis. Ann Neurol 1998;43:499-506.

26. Erskine MK, Cook LL, Riddle KE, et al. Resolution-dependent estimates of multiple sclerosis lesion loads. Can J Neurol Sci 2005;32:205-212.

27. Filippi M, Rovaris M, Capra R, et al. A multi-centre longitudinal study comparing the sensitivity of monthly MRI after standard and triple dose gadolinium-DTPA for monitoring disease activity in multiple sclerosis: implications for phase II clinical trials. Brain 1998;121:2011-2020.

28. Ingle GT, Sastre-Garriga J, Miller DH, Thompson AJ. Is inflammation important in early PPMS? a longitudinal MRI study. J Neurol Neurosurg Psychiatry 2005;76:1255-1258.

29. Molyneux PD, Filippi M, Barkhof F, et al. Correlations between monthly enhanced MRI lesion rate and changes in T2 lesion volume in multiple sclerosis. Ann Neurol 1998;43:332-339.

30. Miller DH, Molyneux PD, Barker GJ, et al. European Study Group on Interferon- $\beta 1 \mathrm{~b}$ in Secondary Progressive Multiple Sclerosis. Effect of interferon- $\beta 1 \mathrm{~b}$ on magnetic resonance imaging outcomes in secondary progressive multiple sclerosis: results of a European multicenter, randomized, double-blind, placebo-controlled trial. Ann Neurol 1999;46:850-859.

31. Jacobs LD, Cookfair DL, Rudick RA, et al. Multiple Sclerosis Collaborative Research Group (MSCRG). Intramuscular interferon $\beta$-1a for disease progression in relapsing multiple sclerosis. Ann Neurol 1996;39:285-294.

32. IFNB Multiple Sclerosis Study Group; University of British Columbia MS/MRI Analysis Group. Interferon $\beta$-1b in the treatment of multiple sclerosis: final outcome of the randomized controlled trial. Neurology 1995;45:1277-1285.

33. Li DK, Paty DW. Prevention of Relapses and Disability by Interferon- $\beta 1$ a Subcutaneously in Multiple Sclerosis. Magnetic resonance imaging results of the PRISMS trial: a randomized, doubleblind, placebo-controlled study of interferon- $\beta 1 \mathrm{a}$ in relapsingremitting multiple sclerosis. Ann Neurol 1999;46:197-206.

34. Filippi M, Horsfield MA, Tofts PS, et al. Quantitative assessment of MRI lesion load in monitoring the evolution of multiple sclerosis. Brain 1995;118:1601-1612.
35. Isaac C, Li DK, Genton M, et al. Multiple sclerosis: a serial study using MRI in relapsing patients. Neurology 1988;38:1511-1515.

36. Willoughby EW, Grochowski E, Li DK, et al. Serial magnetic resonance scanning in multiple sclerosis: a second prospective study in relapsing patients. Ann Neurol 1989;25:43-49.

37. Lee MA, Smith S, Palace J, Matthews PM. Defining multiple sclerosis disease activity using MRI T2-weighted difference imaging. Brain 1998;121:2095-2102.

38. Brex PA, Ciccarelli O, O'Riordan JI, et al. A longitudinal study of abnormalities on MRI and disability from multiple sclerosis. N Engl J Med 2002;346:158-164.

39. Weiner HL, Guttmann CR, Khoury SJ, et al. Serial magnetic resonance imaging in multiple sclerosis: correlation with attacks, disability, and disease stage. J Neuroimmunol 2000;104:164-173.

40. Khoury SJ, Guttmann CR, Orav EJ, et al. Changes in activated T cells in the blood correlate with disease activity in multiple sclerosis. Arch Neurol 2000;57:1183-1189.

41. Bitsch A, Kuhlmann T, Stadelmann C, et al. A longitudinal MRI study of histopathologically defined hypointense multiple sclerosis lesions. Ann Neurol 2001;49:793-796.

42. Bagnato F, Jeffries N, Richert ND, et al. Evolution of T1 black holes in patients with multiple sclerosis imaged monthly for 4 years. Brain 2003;126:1782-1789.

43. Meier DS, Guttmann CR. Time-series analysis of MRI intensity patterns in multiple sclerosis. Neuroimage 2003;20:1193-1209.

44. Meier DS, Guttmann CR. Time-series modeling of MS lesion development. Neuroimage 2006;32:531-537.

45. Lucchinetti C, Bruck W, Parisi J, et al. A quantitative analysis of oligodendrocytes in multiple sclerosis lesions: a study of 113 cases. Brain 1999;122:2279-2295.

46. Lucchinetti C, Bruck W, Parisi J, et al. Heterogeneity of multiple sclerosis lesions: implications for the pathogenesis of demyelination. Ann Neurol 2000;47:707-717.

47. Patrikios P, Stadelmann C, Kutzelnigg A, et al. Remyelination is extensive in a subset of multiple sclerosis patients. Brain 2006; 129:3165-3172.

48. Lassmann H. Recent neuropathological findings in MS-implications for diagnosis and therapy. JNeurol 2004;251(Suppl 4):IV2IV5.

49. Schwartz M, Kipnis J. Protective autoimmunity and neuroprotection in inflammatory and noninflammatory neurodegenerative diseases. J Neurol Sci 2005;233:163-166.

50. van Waesberghe JH, van Walderveen MA, Castelijns JA, et al. Patterns of lesion development in multiple sclerosis: longitudinal observations with T1-weighted spin-echo and magnetization transfer MR. AJNR Am J Neuroradiol 1998;19:675-683.

51. Filippi M, Rovaris M, Rocca MA, et al. Glatiramer acetate reduces the proportion of new MS lesions evolving into "black holes." Neurology 2001;57:731-733. 